\title{
An Investigation of Verbal Short-term Memory and Phonological Processing in Four Children With Williams Syndrome
}

\author{
S. Majerus ${ }^{1}$, K. Barisnikov ${ }^{2}$, I. Vuillemin ${ }^{2}$, M. Poncelet ${ }^{1}$ and M. van der Linden ${ }^{1,2}$ \\ ${ }^{I}$ Department of Cognitive Sciences, University of Liège, Liège, Belgium and ${ }^{2}$ Department of Psychology, University of Geneva, Geneva, \\ Switzerland
}

\begin{abstract}
Although phonological processing is generally considered to be a proficiency in Williams syndrome (WS), there are very few studies which have extensively explored phonological processing abilities in WS. In this study, we re-assessed phonological processing in WS by exploring verbal STM and phonological awareness abilities in 4 children with WS (CA: 10-12 years) and two control groups, one matched for chronological age (CA) and the other matched for verbal mental age (VA). Our results confirm and extend previous claims of preserved phonological STM in WS by showing specifically preserved STM performance for non-words, compared to both VA and CA control groups. However, we observed that this was the case only for non-words where support of phonological and lexico-semantic knowledge was minimized, with reduced phonological and lexico-semantic effects on STM performance. Furthermore, a more direct assessment of phonological processing abilities through phonological awareness tasks showed impaired performance for the $4 \mathrm{WS}$ children. Our data confirm that STM for non-words represents a real strength in WS but they do not support previous assumptions of a more general preservation of phonological processing abilities in WS. Implications for impaired and preserved cognitive processes underlying verbal STM and phonological awareness abilities in WS are discussed.
\end{abstract}

\section{Introduction}

Williams syndrome (WS) is a rare genetically-based neuro-developmental disorder, involving a microscopic deletion of one copy of several contiguous genes on chromosome 7 (Ewart et al., 1994; Frangiskakis et al., 1996). The cognitive profile of WS is quite distinctive, with relative strengths in language and facial processing, and a profound impairment in spatial cognition (Howlin et al., 1998; Jarrold et al., 1998; Bellugi et al., 2000). However, although language competencies are generally described as relatively preserved in WS, this preservation is not necessarily the same for phonological, lexico-semantic and morpho-syntactic aspects of language processing. Phonological processing, which is the primary focus of this study, is often considered to be the best developed aspect of language processing in WS as individuals with WS generally present fluent and well-articulated speech without any phonological or phonetic deformations. Regarding lexico-semantics, children with WS generally tend to have a rich receptive and expressive vocabulary but few adult individuals with WS will attain chronological age-appropriate receptive vocabulary, with less precise and abnormally ordered lexico-semantic representations (e.g. Howlin et al., 1998; Jarrold et al., 1998; Bishop, 1999; Patersonef al., 1999; Mervis and Robinson, 2000; Temple et al., 2002). Individuals with WS also seem to present difficulties for various aspects of morphosyntax, such as use of prepositions, grammatical gender assignment and irregular past-tense formation (e.g. Clahsen and Almazan, 1998; Karmiloff-Smith et al., 1998; Volterra et al., 2001).

Although phonological processing is generally considered to be the best developed aspect of language processing in WS, there is, surprisingly, relatively little direct evidence for this assumption. Only two studies have more explicitly addressed this question. A first study by Böhning et al. (2001) has shown that individuals with WS perform as well as chronological age-matched (CA) controls in identifying and repeating auditorily presented vowel-consonant-vowel (VCV) nonsense syllables (/ $\Lambda \mathrm{ba}: /, / \Lambda \mathrm{va}: /)$, suggesting at least that identification of phonetic information is preserved in WS. A second study by Laing et al. (2001), investigating the relationship between phonological skills and reading development in 15 WS subjects and a control group matched for reading test age, as well as verbal and non-verbal mental age, also assessed phonological awareness abilities in WS. They observed equivalent performance in the WS group and the control group for most measures of phonological awareness (rhyme awareness, spoonerisms) except for phoneme deletion measures, suggesting that phonological awareness abilities might also be preserved in WS. However, the verbal age-matched (VA) control group (mean age: 6 years 9 months; range: 5 years to 9 years 2 months) was much younger than the WS group (mean age: 15 years 1 month; range: 9 years to 27 years 7 months), due to the matching not only of mental verbal age, but also of reading age and non-verbal mental age, which are typically relatively low in WS. This 
makes a comparison of the means between the WS and the control group's phonological awareness measures relatively difficult, especially as the control group with an age range of 5 years to 9 years 2 months included children whose phonological awareness abilities were still relatively poor while others had fully developed phonological awareness abilities, leading to a large variance in the scores measuring these abilities. Also we do not know whether phonological awareness abilities are still preserved in WS individuals when compared to a control group matched for verbal mental age only (which is normally better developed in WS than reading ability) and to a control group matched for chronological age.

Further evidence for a proficiency in phonological processing comes from studies which have investigated phonological processing rather indirectly, by assessing STM performance for verbal information. In STM tasks, verbally presented material of increasing length has to be repeated exactly in the same form as it was presented. Short-term storage of verbal information in these tasks has been interpreted as reflecting the intervention of a phonologically based short-term memory system (Baddeley and Hitch, 1974; Baddeley, 1986; Baddeley et al., 1998). Furthermore, these tasks also require well developed abilities in phonological analysis, identification, segmentation and articulatory planning of the phonological information contained in the auditorily presented verbal information. Several studies have indeed shown a special proficiency in WS for STM tasks using verbal stimuli, which cannot be attributed to a more general preservation of STM processing as STM for visuo-spatial material is severely impaired (Wang and Bellugi, 1994; Barisnikov et al., 1996; Jarrold et al., 1999; Vicari et al., 1996a).

A number of studies have also shown that STM performance in WS individuals is affected by the same phonological variables as in normally developing children: they showed an advantage for short-term recall of phonologically similar versus dissimilar words and for long versus short words (Barisnikov et al., 1996; Vicari et al., 1996b). Phonologically dissimilar words are thought to be represented by more distinctive traces in STM than phonologically similar words and thus are easier to retrieve (Baddeley and Hitch, 1974; Baddeley, 1986). The word length effect has also been interpreted as being related to the phonological characteristics of the items to be recalled: longer words contain more phonological information and thus take more time to be rehearsed in STM or lead to more interference between stimuli due to their greater phonological complexity (Baddeley and Hitch, 1974; Baddeley, 1986; Nairne, 1990; Service, 1998).

Furthermore, Vicari et al. (1996a, b) have shown that in STM tasks, compared to normal mental age-matched controls, the word frequency effect (better recall of high versus low frequency words) is reduced in WS and primacy effects (better recall of the first items in a STM list) are absent. These effects have been interpreted as reflecting the influence of lexico-semantic knowledge on STM tasks (Roodenrys et al., 1994). These results suggest that the preserved performance observed in verbal STM in WS is less related to the influence of lexicosemantic knowledge than in controls and therefore might strongly depend on well developed phonological storage abilities.

The most stringent evidence coming from verbal STM studies for a specific strength in phonological processing in WS should however be derived from STM tasks measuring specifically short-term storage capacities for phonological information like non-words. In fact, evidence for intact short-term retention of non-words in WS is relatively scarce and conflicting. Grant et al. (1996) presented to English-speaking individuals with WS a French and an English non-word repetition task, based on the non-word repetition test devised by Gathercole et al. (1994) for measuring phonological short-term storage capacities. WS individuals showed a significantly poorer performance than mental age-matched English and French control groups in repeating French non-words. According to Grant et al. (1996), the fact that English-speaking WS individuals showed poorer performances in repeating French non-words than mental age-matched French controls and that their performance in repeating English non-words was significantly higher than their performance for repeating French non-words suggests that they have no specific proficiency in the mimicry of foreign sounds, but that the WS subjects probably used their native English phonological representations to support English non-word repetition. However, this does not explain why their performance for French non-word repetition was still poorer than that of mental age-matched English-speaking controls. If phonological short-term storage capacities and support of English phonological representations are preserved in WS, then English-speaking WS and their English-speaking control group should have had a similar performance. But this was not the case and thus suggests that short-term storage of phonological information, as measured by non-word repetition, was not really a strength in the WS group of Grant et al. (1996).

In a second study, Grant et al. (1997) observed that performance on an English non-word repetition task in a group of WS children was indeed below the performance level which might be expected from their verbal mental age, further suggesting that short-term storage of phonological information was not really a strength in this WS 
group. However, it must be noted that the non-words used by Grant et al. (1997) were non-words from the Children's Test of Non-word Repetition (Gathercole and Baddeley, 1996). These non-words have a rather complex multisyllabic structure and contain many grammatical and lexical morphemes (such as "-ually", "ation", "-tory"). As individuals with WS seem to have difficulties with lexically-based grammatical words (e.g. Volterra et al., 2001), it cannot be excluded that the relatively low performance for non-word repetition could be partially explained by difficulties in processing lexical and grammatical morphemes. Indeed, Barisnikov et al. (1996) showed that their WS case CS showed chronological age-appropriate performance in a non-word repetition task which used consonant-vowel (CV) and consonant-consonant-vowel (CCV) non-word syllables containing no grammatical morphemes (Poncelet and Van der Linden, 2003). Clearly, STM for non-words in WS needs further investigation.

To sum up, empirical evidence for a preservation of phonological processing in WS, whether measured by direct or more indirect measures, remains weak and uncertain. The aim of the present study is precisely to provide further empirical data regarding the status of phonological processing abilities in WS, by re-examining phonological STM abilities (Experiment 1) and phonological awareness abilities (Experiment 2) in 4 children with WS, in the light of the methodological issues raised above.

\section{Experiment 1: Verbal STM}

STM performance for both word and non-word stimuli was investigated in order to re-assess whether STM, especially for non-words, is really a strength in WS and thus can be taken as indirect evidence for preserved phonological processing in WS. Contrary to previous studies (e.g. Grant et al., 1996), the non-words used in this study had a simple CV, CCV or CVC structure and did not contain any grammatical morphemes. This was done in order to rule out the possibility that any difficulties in processing grammatical morphemes could contaminate short-term storage of non-words. We also investigated the influence of both phonological - word length and phonological similarity - and lexico-semantic - word frequency - variables on STM performance which had already been explored in previous studies (Vicari et al., 1996a, b). Furthermore, we also explored the influence of variables which have not yet been assessed in WS: non-word phonotactic frequency and lexicality. The lexicality effect is characterized by an advantage in recalling words versus non-words and thus also represents the influence of lexico-semantic knowledge (Hulme et al., 1991). The non-word phonotactic frequency effect represents an advantage in recalling non-words containing frequent versus infrequent phoneme associations and thus represents the influence of phonological knowledge about the statistical properties concerning the structure of the native phonology. Gathercole et al. (1999) and Majerus and Van der Linden (2003) showed that this effect is present in normally developing children aged 6 to 16 years. Grant et al. (1997) claimed that this effect should also be normal in WS, on the basis of normal wordlikeness effects observed in a non-word repetition task for their WS participants. However, in that study, non-word phonotactic frequency was not directly controlled and wordlikeness ratings of the non-words used were only based on wordlikeness judgements obtained from 20 undergraduate students.

We predicted that, if phonological processing abilities are preserved in WS, then verbal STM should be preserved in WS especially for items depending mostly on phonological coding, such as non-words containing no grammatical or lexical morphemes. Furthermore, we should expect a normal influence of phonological variables on STM performance such as word length, phonological similarity and especially phonotactic frequency, while the influence of lexico-semantic variables might be reduced. Contrary to previous studies, we also explored whether the phonological effects are present in each of the WS participants, and not only at the group level (e.g. Vicari et al., 1996b), and we compared their performance to both VA- and CA-matched controls.

\section{Participants}

The Williams syndrome (WS) group comprised four children (3 girls and 1 boy) with an average chronological age of 11 years 4 months (range: 10 years 1 month to 12 years 7 months). Their diagnosis was confirmed genetically (deletion of contiguous genes on chromosome 7, including the genes coding for elastin) and clinically (facial dismorphology, anomalies of the cardiovascular and musculoskeletal system). They presented mental retardation in the mild to moderate range, as evidenced by their performance on the WISC-R intelligence scales (Wechsler, 1981) (average IQ 63, range 53-73), with greater scores on verbal than non-verbal sub-scales (average verbal IQ 77, range 67-86; average performance IQ 55, range 47-66). All four children were in special schools for children with mental retardation and learning difficulties. All children are native French-speakers and are living in the French-speaking community of Switzerland. The four participants are from a middle-class socioeconomic background. 
The performance of the WS children was compared to two control groups. A first control group comprised 12 chronological age-matched (CA) normally developing children (6 girls and 6 boys), with an average age of 11 years 4 months (range: 10 years 4 months to 12 years 9 months). A second control group was a younger control group of normally developing children matched for receptive vocabulary age, using the French version of the PPVT-R vocabulary scales (Dunn et $\alpha$ l., 1993) (see Table 1) and comprised 8 younger children with an average age of 9 years 5 months (range: 7 years 2 months to 12 years 1 month). All children were native Frenchspeakers, living in the French-speaking community of Switzerland and matched as to the socio-economic background to the WS children.

Children were tested individually, and standardized instructions were used for each task. Informed consent was obtained from the WS and the control subjects as well as from their families according to the declaration of Helsinki.

Table 1: Chronological age and vocabulary scores (EVIP; Dunn et al., 1993) of the Williams syndrome (WS) children, the chronological age-matched (CA) control group and the verbal age-matched (VA) control group

\begin{tabular}{lll}
\hline & Chronological age & EVIP - Raw scores \\
\hline WS1 & $11 ; 05$ & 117 \\
WS2 & $11 ; 02$ & 100 \\
WS3 & $12 ; 07$ & 122 \\
WS4 & $10 ; 01$ & 94 \\
CA control group - mean & $11 ; 04$ & 138.83 \\
(range) & $(10 ; 04-12 ; 09)$ & $(114-156)$ \\
VA control group - mean & $9 ; 05$ & 108.5 \\
(range) & $(7 ; 02-12 ; 01)$ & $(86-133)$ \\
\hline
\end{tabular}

\section{Material}

Word length effect

Two sets of eight monosyllabic and eight 3-4 syllabic words were selected (Masquelier, 1989). They were matched for word frequency and imageability. The short and long words were randomly assigned to sequences ranging from 2 to 7 words, with 2 trials per sequence length. The sequences for the short and long word sets were presented in ascending order for immediate serial recall; testing was stopped when the two trials of a given sequence length were incorrectly recalled. Short and long word span were determined by taking as a span measure the length of the last sequence where at least one trial was correctly recalled.

\section{Phonological similarity effect}

Two sets of 8 phonologically similar and 8 phonologically dissimilar words were selected (Masquelier, 1988). The words were all monosyllabic and matched for word frequency and word imageability. The two word sets were randomly assigned to sequences ranging from 2 to 7 words, with 2 trials per sequence length. The procedure was the same as for short and long word lists, and word span for phonologically similar and dissimilar word sets was determined.

\section{Non-word repetition}

Phonological STM was also assessed by using a non-word repetition task (Poncelet and Van der Linden, 2003). This task was designed in such a way as to rule out as much as possible long-term memory contribution to phonological short-term memory performance (see Gathercole and Baddeley, 1989). It consisted of 36 nonwords: 21 items composed of CV syllables (3 non-words each containing 2, 3, 4, 5, 6, 7 and 8 syllables) and 15 items composed of CCVsyllables ( 3 non-words each containing 2, 3, 4, 5, and 6 syllables). None of the syllables corresponded to any morpheme in French. However, the phoneme sequences in each non-word conformed to the phonotactic rules of French. Each non-word was spoken by the experimenter. The set of CCV items followed the set of CV items and in each set the items were presented in ascending order. The subject had to listen and then to repeat immediately each item; this was recorded on an audio cassette recorder. A response was scored as incorrect if it differed phonemically from the target non-word. However, one transformation of one articulatory 
feature was considered as correct. The total number of items and syllables correctly recalled was determined separately for CV and CCV non-words.

\section{Word frequency effect}

The influence of lexical long-term knowledge on STM performance was assessed by presenting lists of lexically frequent (HF) and infrequent (LF) word lists for immediate serial recall. Two lists of 108 words were constructed. The items in the two lists were matched for item length and were all bisyllabic. The frequency count was $<200$ and $>10000$, for the HF and LF lists, respectively (Content et al., 1990). The total number of words correctly recalled and in correct serial position, summed over all sequence lengths, was determined for the HF and LF word lists.

\section{Lexicality and non-word phonotactic frequency effects}

In order to assess lexicality and non-word phonotactic frequency effects in STM, 3 categories of monosyllabic stimuli were created: 80 low phonotactic frequency non-words (LF non-words) (examples: /dog/, /zug/, /dz̃z/,, /nov/, /fø $\mathrm{f} /$, /poz/, /mug/, /sog/, /tof/, /bãm/), 80 high phonotactic frequency non-words (HF non-words) (examples: /foz/, /buv/, /dz̃p/, /toR/, /nœz/, /møv/, /Ruz/, /gov/, /pok/, /tãn/), and 80 words (examples: taupe [mole], tour [tower], dinde [turkey], sauce [sauce], jeune [youngster], nord [north], route [road], pause [stop], faute [error], danse [dance]). Each stimulus had the same syllabic structure $\left(\mathrm{C}_{1} \mathrm{VC}_{2}\right)$. Phonotactic frequency was established using the Phonetic Database of French by Tubach and Boë (1990). This database was constructed by transcribing in phonetics formal and informal conversations in French; frequency of co-occurrence of phonemes, diphones, and triphones was then calculated on the basis of this oral language corpus. This corpus maximises the validity of the phonotactic frequency counts for tasks using auditory verbal stimuli as the counts are based on real spoken language, and not on written language as was the case for phonotactic frequency counts used in previous studies (e.g. Vitevitch and Luce, 1998; Gathercole et al., 1999). We used the diphone frequency counts as a measure of phonotactic frequency. The LF non-words were constructed using $\mathrm{C}_{1} \mathrm{~V}$ and $\mathrm{VC}_{2}$ diphones which are relatively rare in French (e.g. in the non-word /dog/, the diphones /do/ and /og/ have a frequency of 124 and 31 , respectively). The $\mathrm{HF}$ non-words contained $\mathrm{C}_{1} \mathrm{~V}$ and $\mathrm{VC}_{2}$ diphones which are significantly more frequent in French; for example, in the non-word /foz/, the diphones /fo/ and /oz/ have a frequency of 236 and 553, respectively. Mean phonotactic frequency for $\mathrm{HF}$ non-words was $660\left(\mathrm{C}_{1} \mathrm{~V}\right)$ and $964\left(\mathrm{VC}_{2}\right)$. Mean phonotactic frequency for $\mathrm{LF}$ non-words was $160\left(\mathrm{C}_{1} \mathrm{~V}\right)$ and $120\left(\mathrm{VC}_{2}\right)$; the difference in diphone frequency between the $\mathrm{HF}$ non-words and LF non-words was significant for the $\mathrm{C}_{1} \mathrm{~V}$ diphones $[\mathrm{t}(1,222)=7.94, \mathrm{P}<.0001]$ and the $\mathrm{VC}_{2}$ diphones $[t(1,222)=11.35, \mathrm{P}<.0001]$. Finally, the list of words used in this experiment was created by selecting monosyllabic frequent and imageable words whose diphone frequencies were matched to those of the HF nonwords (mean diphone frequency for the words: $695\left(\mathrm{C}_{1} \mathrm{~V}\right)$ and $\left.1021\left(\mathrm{VC}_{2}\right)\right)$. There was no difference in phonotactic frequency between words and $\mathrm{HF}$ non-words $\left[\mathrm{t}(1,222)<1\right.$ for $\mathrm{C}_{1} \mathrm{~V}$ and $\mathrm{VC}_{2}$ diphones]. The consonants and vowels used for the LF non-words, HF non-words and words were sampled from the same pool of phonemes. Each word and non-word was used only once in the experiment.

The word, HF non-word and LF non-word stimuli were regrouped in sequences of increasing length for the ISR task; the shortest sequence contained 2 stimuli and the longest sequence contained 6 stimuli. There were 4 trials for each stimulus type at each list length. Children were presented the non-word and word sequences for immediate serial recall. For each sequence length, the 4 trials for the word, HF non-word and LF non-word stimuli were presented before going on to the next sequence length. The order of presentation of word, HF nonword and LF non-word sequences was randomized between subjects. The participants were asked to repeat the sequences immediately after auditory presentation by the examiner. Responses of subjects were recorded on tape and transcribed for later scoring. For the words, HF non-word and LF non-word lists, all stimuli that had been correctly repeated and in correct serial position, across all sequence lengths, were scored. As a measure of STM performance, the total number of correctly recalled items and in correct serial position, for the words, LF nonword and HF non-word lists, was counted.

The different STM tasks and conditions were presented in randomized order between the different subjects in order to rule out that any differences between the different stimulus conditions could be an artefact of the order of presentation of the different tasks.

\section{Results and discussion}

Impairment of the WS children's performance was determined by computing modified t-tests (Crawford and Garthwaite, 2002) on each child's score compared to performance in the CA and VA control groups. As shown in Table 2, the 4 WS children performed in the normal range for the vast majority of the different STM tasks. 
Only long word span was significantly below the mean performance of CA-matched controls in all 4 WS children. A word length effect was observed in 3 out of the 4 WS children. A phonological similarity effect was observed in 2 out of the $4 \mathrm{WS}$ children. Most remarkably, the 4 WS children presented quite preserved performances in non-word repetition, with equal or even better, but not significantly better, performances than the VA-matched control group. This suggests that short-term storage of verbal information, and especially for phonological information which is devoid of any long-term language representations, may be a specific proficiency in WS.

The results of the influence of long-term phonological and lexico-semantic knowledge on STM performance are reported in Table 3. We observed that, in the STM task assessing phonotactic frequency and lexicality effects, performance was impaired in $3 \mathrm{WS}$ children for short-term retention of words and HF non-words, compared to both control groups. In contrast, the performance was in the normal range for short-term storage of LF nonwords for all four WS children, compared to the VA control group, and impaired in only two WS children compared to the CA control group. In the STM task assessing the word frequency effect, performance was impaired in $3 \mathrm{WS}$ children for short-term retention of HF words, while performance for the LF words was normal for all the WS children, except for WS4 whose performance was impaired compared to both control groups.

Table 2: Results for the STM tasks measuring word length and phonological similarity effects, and for non-word repetition, for each WS participant, and the CA and VA control groups

\begin{tabular}{|c|c|c|c|c|c|c|c|c|}
\hline & $\begin{array}{l}\text { Span: } \\
\text { long } \\
\text { words }\end{array}$ & $\begin{array}{l}\text { Span: } \\
\text { short } \\
\text { words }\end{array}$ & $\begin{array}{l}\text { Span: } \\
\text { phon. } \\
\text { similar } \\
\text { words }\end{array}$ & $\begin{array}{l}\text { Span: phon. } \\
\text { dissimilar } \\
\text { words }\end{array}$ & $\begin{array}{l}\text { NWR } \\
\text { (CV): } \\
\text { items } \\
\text { correct }\end{array}$ & $\begin{array}{l}\text { NWR (CV): } \\
\text { syllables } \\
\text { correct }\end{array}$ & $\begin{array}{l}\text { NWR } \\
\text { (CCV): } \\
\text { items } \\
\text { correct }\end{array}$ & $\begin{array}{l}\text { NWR } \\
\text { (CCV): } \\
\text { syllables } \\
\text { correct }\end{array}$ \\
\hline WS1 & $3 *$ & 4 & 3 & 4 & 13 & 71 & 8 & 39 \\
\hline WS2 & $3 *$ & 4 & 3 & 5 & 13 & 62 & $7 *$ & 31 \\
\hline WS3 & $3 *$ & 4 & 4 & 4 & 14 & 80 & 10 & 42 \\
\hline WS4 & $3 *$ & $3^{*, a}$ & 3 & $3 *$ & 11 & $55^{*}$ & $7 *$ & 31 \\
\hline \multicolumn{9}{|l|}{ CA } \\
\hline Mean & 4.5 & 5.08 & 4.33 & 5.42 & 16.08 & 88.25 & 9.83 & 43.5 \\
\hline SD & 0.6 & 0.67 & 1.23 & 1.16 & 3.12 & 14.51 & 1.47 & 8.6 \\
\hline \multicolumn{9}{|l|}{ VA } \\
\hline Mean & 3.75 & 4.5 & 3.62 & 4.87 & 11.5 & 65.62 & 7.75 & 31.5 \\
\hline SD & 0.46 & 0.53 & 0.74 & 1.13 & 3.02 & 17.80 & 2.05 & 5.21 \\
\hline
\end{tabular}

* Difference significant at $\mathrm{p}<.05$ compared to mean scores obtained in CA controls (modified t-test; Crawford and Garthwaite, 2002). ${ }^{a}$ Difference significant at $\mathrm{p}<.05$ compared to mean scores obtained in VA controls.

NWR: non-word repetition for non-words with a simple consonant-vowel structure (CV) and for non-words with a complex consonantconsonant-vowel structure (CCV).

Table 3: Results for the STM measures assessing the phonotactic frequency and word frequency effects, for each WS participant, and the CA and VA control groups

\begin{tabular}{llllll}
\hline & Words & HF non-words & LF non-words & Words: High frequency & Words: Low frequency \\
\hline WS1 & $24^{*, a}$ & $18^{*, a}$ & $18^{*}$ & $25^{*, a}$ & 22 \\
WS2 & $20^{*, a}$ & $18^{*, a}$ & $17^{*}$ & 33 & 21 \\
WS3 & 29 & $21^{\mathrm{a}}$ & 25 & $31^{*}$ & 28 \\
WS4 & $25^{*, a}$ & $16^{*, a}$ & 25 & $13^{*, a}$ & $8^{*, a}$ \\
CA & & & & & 43.08 \\
$\quad$ Mean & 44.00 & 32.13 & 26.25 & 57.42 & 12.80 \\
SD & 8.88 & 6.31 & 3.01 & 12.46 & 34.00 \\
VA & & & & & 7.82 \\
$\quad$ Mean & 33.14 & 29.75 & 21.85 & 49.50 & 11.65 \\
SD & 3.58 & 3.72 & 2.73 & &
\end{tabular}

*Difference significant at $\mathrm{p}<.05$ compared to mean scores obtained in CA controls (modified t-test; Crawford and Garthwaite, 2002).

${ }^{a}$ Difference significant at $\mathrm{p}<.05$ compared to mean scores obtained in VA controls. HF non-words: high phonotactic frequency non-words. LF non-words: low phonotactic frequency non-words. 
Significant lexicality $\left(\mathrm{CA}: \mathrm{F}\left(1,7^{1}\right)=48.48\right.$, $\mathrm{p}<.001$; VA: $\left.\mathrm{F}(1,6)=29.4, \mathrm{p}<.01\right)$, phonotactic frequency $(\mathrm{CA}: \mathrm{F}(1,7)$ $=9.82, \mathrm{p}<.05 ; \mathrm{VA}: \mathrm{F}(1,6)=11.11, \mathrm{p}<.05)$ and word frequency effects $(\mathrm{CA}: \mathrm{F}(1,11)=61.26, \mathrm{p}<.001 ; \mathrm{VA}: \mathrm{F}(1,7)$ $=57.00, \mathrm{p}<.001)$ were obtained in both control groups. Regarding the lexicality effect in the WS children, the 4 WS children presented an advantage of 6,2,8, and 9 items, respectively, for recall of words compared to recall of HF non-words, while this advantage was greater in controls, especially when considering the CA control group (CA control group: $\mathrm{M}=11.87, \mathrm{SD}=4.82$, range 4-19; VA control group: $\mathrm{M}=7.00, \mathrm{SD}=3.42$; range 4-14). Modified t-tests computed on difference scores, which were obtained by subtracting performance for nonwords from performance for words, showed that the lexicality effect was significantly smaller only for WS2 $(\mathrm{p}<.05)$, compared to the CA control group. The results are clearer when considering the advantage of word recall compared to LF non-word recall: here the advantage for the WS children was only 6, 3, 4 and 0 items respectively, while the mean advantage was 17.75 (SD: 6.39; range:12-27) for the CA control group and 11.29 (SD: 2.5; range: 8-15) for the VA control group. Modified t-tests showed that the lexicality effect was significantly smaller in all WS children compared to the VA controls $(\mathrm{p}<.05)$, and in all children WS except WS1 compared to CA controls $(\mathrm{p}<.05)$. Regarding the phonotactic frequency effect, the WS children showed either no effect or an inversed phonotactic frequency effect, with an advantage of $0,1,-4$ and -9 items for HF non-words, while the mean advantage was 5.87 (SD: 5.3; range: $-3^{2}-15$ ) in the CA control group and 4.29 (SD: 3.4; range: 1-9) in the VA control group. Modified t-tests showed that the phonotactic frequency effect was significantly smaller in WS3 and WS4 compared to the VA controls $(p<.05)$, and in WS4 compared to CA controls $(\mathrm{p}<.05)$. Finally, the word frequency effect was also somewhat reduced in the WS children: their advantage in recalling HF over LF words was 3, 12, 3 and 5, respectively, while the mean advantage was 14.9 (SD: 6.32; range: 5-24) in the CA control group and 15.5 (SD: 5.81; range: 9-24) in the VA control group. Modified t-tests showed that the frequency effect was significantly smaller in WS1 and WS3 compared to the VA controls $(\mathrm{p}<.05)$

In agreement with previous studies, the results of our STM experiments globally confirm that verbal STM performance can indeed be a strength in WS. In addition to previous results, our study shows that performance can be in the normal range not only compared to VA-matched control groups, but also to CA-matched control groups. However, our study also shows that this is only the case when STM is assessed in a manner where no long-term memory contribution is possible. For example, STM span was relatively normal for the tasks assessing word length and phonological similarity effects. These tasks indeed minimize the retention of item information, and hence the possible influence of language knowledge which operates on storage of item information (Poirier and Saint-Aubin, 1996), while they maximize the retention of serial position information: in these tasks, the same items are presented repeatedly across the different trials, and after a few trials, the subjects might anticipate which items will occur; however, they cannot anticipate in what serial position they will occur and it is precisely this information which has to be retained in STM in these tasks. Furthermore, their performance was also normal in the STM tasks using non-words, and especially when using LF non-words where the influence of phonological and lexical knowledge is less likely. This is also supported by the reduced lexicality, phonotactic frequency and word frequency effects in the WS children, suggesting that language knowledge does not support STM performance to the same extent as it does in controls.

More generally, our data suggest that the relative preservation of non-word STM observed in this and other studies does not necessarily represent evidence for a preservation of phonological language knowledge or a phonological processing factor. Indeed phonological factors such as word length and phonological similarity did not consistently influence STM performance in our $4 \mathrm{WS}$ subjects. However, it has to be noted that these effects are not always consistent in normal subjects either; for example, Logie et al. (1996) showed that these effects can also be absent in normal subjects. More importantly, the phonotactic frequency effect which was consistently observed in our CA- and VA-matched control subjects, but was strongly reduced or even inversed in the four WS children, further suggests that phonological language knowledge influences less consistently their performance in phonological STM tasks. However, another possible hypothesis is that phonological processing itself is not completely normal in our WS children and thus cannot normally influence STM performance. This hypothesis was more directly explored in the next experiment where we explored the preservation of phonological awareness abilities.

\footnotetext{
${ }^{1}$ For the STM tasks assessing lexicality and phonotactic frequency effects, control data were obtained for 8 CA-matched controls and 7 VAmatched controls.

21 control subject presented an inversed phonotactic frequency effect.
} 


\section{Experiment 2: Phonological awareness}

In Experiment 2, the preservation of phonological processing abilities was tested more directly, by exploring phonological awareness abilities in our WS subjects. These abilities have indeed been very rarely explored. Furthermore, contrary to the only study so far which has explored these abilities (Laing et al., 2001), the WS children were compared to both a CA-matched control group and a slightly younger VA-matched control group. This was done in order to compare the WS children's phonological awareness performance to that of control groups with more fully developed and homogeneous phonological awareness abilities. Similarly to Experiment 1 , the influence of phonological and lexico-semantic variables on performance on phonological awareness tasks was assessed by measuring non-word phonotactic frequency and lexicality effects, which has not been done in any previous studies. Phonological awareness (PA) was explored in tasks which have typically been used in developmental studies to explore phonological analysis and segmentation abilities (Morais et al., 1987). We assessed PA at three different levels: (1) word-, (2) rhyme-, and (3) phoneme-level, using minimal pair discrimination, rhyme judgement and phoneme detection tasks.

\section{Method}

\section{Material}

PA at the word-level was assessed with a minimal pair discrimination task, in which pairs of words were presented differing (or not) by a single phoneme. This judgement can be realized by comparing the global word forms of the two items of a pair, without being aware of the precise phoneme which differentiates the two words. Therefore, this is the easiest PA task and is quite accurately performed by very young children.

PA at the rhyme-level was assessed with a rhyme judgement task, in which pairs of words were presented with similar or different rhymes. The detection of rhyme requires the segmentation of the word forms in onset, nucleus and rhyme. This judgement cannot be correctly made on the basis of global word form comparison as the two words of each pair are always different.

PA at the phoneme-level was assessed with a phoneme detection task, in which a previously presented consonant had to be detected in the onset of a presented word. This task requires the segmentation of the word onset in phonemes which have to be compared with a target phoneme. This task is more difficult and is typically acquired later than minimal pair discrimination and rhyme judgement.

In each task, phonological awareness was assessed for three different stimulus types: words, non-words composed of diphones with a high probability of occurrence in French (HF non-words) and non-words composed of diphones with a low probability of occurrence (LF non-words). This was done in order to assess whether a better performance in phonological awareness tasks could be observed for words compared to high phonotactic frequency non-words, and for high compared to low phonotactic frequency non-words. If this is the case, it would suggest that phonological and lexico-semantic knowledge about the items to be processed facilitates phonological judgements.

The minimal pair discrimination task consisted of 10 word pairs, $10 \mathrm{HF}$ non-word pairs, and $10 \mathrm{LF}$ non-word pairs. The word and non-word stimuli were all unisyllabic and had a $\mathrm{C}_{1} \mathrm{VC}_{2}$ structure. For each stimulus condition, 5 pairs were identical and 5 pairs differed at the level of the first or last consonant by one or two articulatory features (e.g. gomme [gum] - comme [like] for words, /gon/ - /fon/ for HF non-words, /gyf/ - /myf/ for LF non-words). Phonotactic frequency of phoneme associations contained in the non-words was established according to the Phonetic Database of French by Tubach and Boe (1990), as in Experiment 1. There were no differences in diphone frequency between the words and the HF non-words ( 351 vs. 319 for $\mathrm{C}_{1} \mathrm{~V}$ diphones; 575 vs. 575 for $\mathrm{VC}_{2}$ diphones). HF non-words and $\mathrm{LF}$ non-words differed significantly in diphone frequency: 319 vs. $87[\mathrm{t}(19)=-5.192, \mathrm{P}<.0001]$ for $\mathrm{C}_{1} \mathrm{~V}$ diphones, and 561 vs. $110[\mathrm{t}(19)=-4.669, \mathrm{P}<.0001]$ for $\mathrm{VC}_{2}$ diphones.

The rhyme judgement task also consisted of 10 word pairs, $10 \mathrm{HF}$ non-word pairs, and $10 \mathrm{LF}$ non-word pairs. For each stimulus condition, 5 pairs shared the rhyme (e.g. masse [volume] - tasse [cup] for words, /sav/ - /nav/ for HF non-words, /pãm/ - /fãm/ for LF non-words) and 5 pairs did not share the rhyme (e.g. page [page] femme [woman] for words, /mam/ - /tad/ for HF non-words, /mub/ - /tug/ for LF non-words). The word and nonword stimuli were once again unisyllabic and had $\mathrm{C}_{1} \mathrm{VC}_{2}$ structure. There were no differences in diphone frequency between the words and the HF non-words ( 905 vs. 806 for $\mathrm{C}_{1} \mathrm{~V}$ diphones; 1036 vs. 863 for $\mathrm{VC}_{2}$ diphones). HF non-words and LF non-words differed significantly in diphone frequency: 806 vs. 259 [t(19) = $3.349, \mathrm{P}<.01]$ for $\mathrm{C}_{1} \mathrm{~V}$ diphones, and 863 vs. 129 [t(19) = -5.563, $\mathrm{P}<.0001$ ] for $\mathrm{VC}_{2}$ diphones. 
The phoneme detection task consisted of 10 words, $10 \mathrm{HF}$ non-words and $10 \mathrm{LF}$ non-words. For each stimulus condition, half of the items were followed by a target consonant which coincided with the first consonant of the item, and half were followed by a non-matching target consonant. For each stimulus condition, the consonants probed were $/ \mathrm{g} /, / \mathrm{f} /, / \mathrm{b} /, / \mathrm{l} /, / \mathrm{m} /$ and $/ \mathrm{d} /$. The word and non-word stimuli were again unisyllabic and had a $\mathrm{C}_{1} \mathrm{VC}_{2}$ structure (e.g. fête [party] for words, /fєm/ for HF non-words, /fãp/ for LF non-words). There were no differences in diphone frequency between the words and the HF non-words ( 587 vs. 587 for $\mathrm{C}_{1} \mathrm{~V}$ diphones; 1065 vs. 899 for $\mathrm{VC}_{2}$ diphones). HF non-words and LF non-words differed significantly in diphone frequency: 587 vs. $106[\mathrm{t}(9)=$ $-3.33, \mathrm{P}<.01]$ for $\mathrm{C}_{1} \mathrm{~V}$ diphones, and 899 vs. $135[\mathrm{t}(9)=-3.87, \mathrm{P}<.01]$ for $\mathrm{VC}_{2}$ diphones.

\section{Procedure}

The minimal pair discrimination, the rhyme judgement and the phoneme detection task, as well as the different stimulus conditions, were presented in randomized order among the different participants. For each task, first the word list was presented because pilot data had shown that it was sometimes difficult for children to comprehend the task requirements if they had to begin with a non-word list. Then the HF non-word and LF non-word lists were presented in a blocked design. Order of presentation of the 2 non-word lists was randomized between subjects. Each task was preceded by 3 practice items. For the minimal pair discrimination task, the children were told: "You will now hear words (for non-words: funny words you do not know). You will hear two of them presented together each time, and I ask you to tell me if the two words are the same or different"; for the rhyme judgement task, the children were told: "You will once again hear words (for non-words: funny words you do not know). Once again, you will hear two of them presented together each time, but this time, I ask you to tell me if the two words have the same sounds at the end or not."; for the phoneme detection task, the children were told: "You will hear words (for non-words: funny words you do not know). After each word, I will give a sound, and I will ask you to tell me if, yes or no, the word begins with the sound I gave you just after the word". The stimuli were presented orally by the experimenter. Children replied by a simple "yes-no" answer and the number of correct responses within each task and each stimulus condition was noted. The participants were the same as in Experiment 1 .

\section{Results and discussion}

As in Experiment 1, impairment of the WS children's performance was determined by computing modified ttests (Crawford and Garthwaite, 2002) on each child's score compared to performance in the CA and VA control groups. As shown in Table 4, performance for the 4 WS children was significantly impaired for most phonological awareness measures, compared to both control groups. However, it is also obvious that there was some degree of variability in the performance profile for the 4 WS children. While WS1, WS2 and WS4 were impaired on 6,8 , and 6 measures out of 9 , respectively, WS3 was impaired only on 4 measures out of 9, with perfect scores in the phoneme detection task.

Regarding the different stimulus conditions, no consistent differences were observed between words, HF and LF non-words, in the CA and VA control groups. This absence of differential performance between the different stimulus conditions is best explained by the overall ceiling performance in controls for the three stimulus conditions in each of the three tasks. However, regarding our WS subjects, their performances were certainly not at ceiling level; nevertheless, there was no reliable advantage for words over HF non-words, and no advantage for HF over LF non-words. These results are similar to those observed for the STM performance, where WS children also did not present the typical phonotactic frequency effects and a reduced lexicality effect. These data suggest that phonological awareness abilities are not really preserved, at least in 3 out of the 4 WS children. Furthermore phonological and lexico-semantic language knowledge do not seem to influence performance in phonological awareness tasks.

This raises the question whether other factors such as attentional difficulties could explain the results we have obtained. Indeed, some studies have shown that WS subjects may present reduced attentional capacities (Laakmann, 2001; Sherrets et al., 1982). The variability in the performance profiles we observed could indeed be related to distraction and difficulties in maintaining focused attention. However, it must be noted that the STM tasks were also demanding at the attentional level, and especially the STM tasks using non-word stimuli. The fact that all four WS children had relatively normal performance in the STM tasks that were most demanding at the attentional level suggests that possible attentional difficulties are not the whole story in accounting for the difficulties on the phonological awareness tasks. Furthermore, in the minimal pair discrimination task, probably the phonological awareness task which was the least demanding at the attentional level, performance was not better than in the phoneme detection task which was more demanding in attentional resources. A final argument against the possibility that attentional difficulties could explain the performance profile for the phonological 
awareness tasks is that the stimuli used in the STM tasks assessing the lexicality and the phonotactic frequency effect and those used in the phonological awareness tasks were equivalent in phonological complexity and had been constructed following the same principles. Thus, in order to correctly repeat two, three or more word or non-word items in the STM task, attentional demands needed to perceive, analyze and segment the item in order to ensure correct repetition are at least the same as those needed to perform the same operations in the minimal pair discrimination task, where only two words or non-words are presented on each trial and where simply a same-different judgement has to be made. We, therefore, believe that possible attentional difficulties cannot completely explain the impairment in the phonological awareness tasks we observed in three of our WS children

Table 4: Number of correct responses in the three phonological awareness tasks for each WS subject as well as mean of correct responses for the chronological age-matched (CA) and the verbal age-matched (VA) control groups, as a function of stimulus type (HF non-words: high phonotactic frequency non-words; LF non-words: low phonotactic frequency non-words)

\begin{tabular}{|c|c|c|c|c|c|c|c|c|c|}
\hline & \multicolumn{3}{|c|}{$\begin{array}{l}\text { Minimal pair } \\
\text { discrimination }\end{array}$} & \multicolumn{3}{|c|}{ Rhyme judgement } & \multicolumn{3}{|c|}{ Phoneme detection } \\
\hline & Words & $\begin{array}{l}\text { HF non- } \\
\text { words }\end{array}$ & $\begin{array}{l}\text { LF non- } \\
\text { words }\end{array}$ & Words & $\begin{array}{l}\text { HF non- } \\
\text { words }\end{array}$ & $\begin{array}{l}\text { LF non- } \\
\text { words }\end{array}$ & Words & $\begin{array}{l}\text { HF non- } \\
\text { words }\end{array}$ & LF non-words \\
\hline WS1 & $6^{*, a}$ & 9 & $7 *, \mathrm{a}$ & $5^{*, a}$ & 7 & 8 & $6^{*, a}$ & $5^{*, a}$ & $5^{*, a}$ \\
\hline WS2 & $6^{*, \mathrm{a}}$ & $7 *$,a & $7^{*, \mathrm{a}}$ & $8^{*, a}$ & 7 & $6^{*, a}$ & $5^{*, \mathrm{a}}$ & $6^{*, a}$ & $7 *$, a \\
\hline WS3 & $8 *, a$ & $7^{*, a}$ & 10 & 9 & $4^{*, \mathrm{a}}$ & $7^{*, a}$ & 10 & 10 & 10 \\
\hline WS4 & 10 & $8^{*, \mathrm{a}}$ & $8^{*, a}$ & $7 *, \mathrm{a}$ & 7 & $7^{*}$, a & $2^{*, \mathrm{a}}$ & 10 & $7^{* \text {,a }}$ \\
\hline \multicolumn{10}{|l|}{ CA } \\
\hline Mean & 10 & 9.75 & 9.92 & 9.67 & 9.08 & 9.25 & 9.83 & 9.42 & 10 \\
\hline SD & 0.00 & 0.45 & 0.29 & 0.49 & 1.38 & 0.75 & 0.39 & 0.67 & 0.00 \\
\hline \multicolumn{10}{|l|}{ VA } \\
\hline Mean & 9.88 & 9.50 & 9.75 & 9.62 & 8.63 & 9.00 & 9.75 & 9.38 & 9.88 \\
\hline SD & 0.35 & 0.53 & 0.46 & 0.52 & 1.30 & 0.93 & .46 & 0.74 & 0.35 \\
\hline
\end{tabular}

*Difference significant at $\mathrm{p}<.05$ compared to mean scores obtained in CA controls (modified t-test; Crawford and Garthwaite, 2002) ${ }^{a}$ Difference significant at $\mathrm{p}<.05$ compared to mean scores obtained in VA controls.

Why did we observe impaired performance in phonological awareness tasks while Laing et al. (2001) did not show such deficits? As we have already mentioned, the verbal age-matched control group (mean age: 6 years 9 months) in the study by Laing et al. (2001) was much younger than ours (mean age: 9 years 5 months). Furthermore, there was a large variability in phonological awareness scores in their control group as it included children aged 5 years when phonological awareness abilities are still not well developed and children aged 9 years 2 months who normally present fully developed phonological awareness abilities, thus making any comparison between their WS group (mean age: 15 years 1 month) and their control group difficult. Our study then shows that when comparing WS children to a slightly younger verbal mental age-matched control group with more homogeneous phonological awareness abilities, then a deficit in phonological awareness in WS appears. However, as these results are derived from a multiple case study approach based on only 4 children with WS aged 10-12 years, they need to be replicated in other studies with different age groups before they can be generalized to the whole WS population.

\section{General discussion}

We investigated phonological processing abilities in WS by assessing performance for verbal STM and phonological awareness tasks. In agreement with previous studies, our $4 \mathrm{WS}$ children presented a proficiency in verbal STM processing, as their performances in the STM tasks, especially for non-words, were in the normal range. However, we observed that this was the case not only compared to a VA control group (as has already been shown), but also compared to a CA control group. In line with previous studies also, the influence of lexico-semantic variables on STM performance, measured in our study by both lexicality and word frequency effects, was reduced. However, phonological effects, measured by word length and phonological similarity effects, but also by non-word phonotactic frequency effects, were also inconsistent or reduced. Furthermore, the preserved performance in non-word STM tasks contrasted with an impairment in phonological awareness tasks, compared to both control groups. The latter results do not support the assumption of well developed phonological processing abilities in WS which has been commonly advanced, but very rarely extensively explored. 
How can we now explain the dissociation between relatively preserved STM performance for non-words but impaired performance in the phonological awareness tasks? At the most basic level, both tasks require intact analysis, identification and segmentation of the auditorily presented verbal information which was preserved, as shown by normal non-word repetition performance in the STM tasks. However, these basic perceptualphonological processes, although necessary, might not be sufficient for correct performance in phonological awareness tasks. Indeed, phonological awareness tasks also require explicit conscious awareness of the segmented phonological information on which explicit phonological judgements have to be made (Morais et al. 1987). This explicit metaphonological awareness might be specifically impaired in WS. However, in other tasks where phonological information also has to be explicitly accessed and retrieved, such as in receptive vocabulary tasks, much better performance is observed (e.g. Howlin et al., 1998). Nevertheless, in phonological awareness tasks, more detailed phonological information has to be consciously accessed and monitored, while in receptive vocabulary tasks partial conscious access to a more global phonological representation of a word might be sufficient to guide selection of the correct response. Thus WS might be characterized by a relatively task-specific impairment in explicit metaphonological awareness. Alternatively, there might be an impairment at the level of the phonological representations themselves, characterized by a poorer or abnormally structured network of phonological representations in long-term memory and/or containing fewer finely-grained phonological representations than in normally developing children. Finely-grained phonological representations are indeed necessary for correct performance in phonological awareness tasks. This possibility is clearly supported by the absence of phonotactic frequency effects, a rather implicit measure reflecting the organization of the phonological network in terms of sublexical phonological representations, in both phonological awareness and STM tasks. This interpretation is further strengthened by data showing that the lexical network in WS seems to be structured in an abnormal way, as individuals with WS seem to produce an abnormally high rate of low frequency words in word fluency tasks and show an abnormal ordering of semantically-related items (Bellugi et al., 1994; Jarrold et al., 2000; Temple et al., 2002). The diphone frequency counts used in our study indeed depended on both the number of different words in which these diphones occur, but also on the frequency of occurrence of the words containing these diphones (Tubach and Boe, 1990). Thus, if the lexical representations are abnormally organized in the language network, then it is likely that sublexical phonotactic representations are also abnormally organized. The reduced lexicality and word frequency effects observed in the STM tasks represent further independent support for this suggestion.

How can we now interpret the reduction of phonological influences on STM performance while at the same time STM performance was particularly preserved for non-words, a measure we considered as our purest measure of phonological processing in STM? Firstly, it may be simply that the verbal short-term storage capacities themselves are intact, but interact less with language knowledge stored in long-term memory than in normal children. This could lead to relatively normal performance for storage of verbal items where a possible support from language representations stored in long-term memory will be reduced (as was the case for our low diphone frequency non-words), but to impaired performance for storage of verbal information depending more on longterm memory support such as words or high diphone frequency non-words. Secondly, as we have discussed above, phonological and lexico-semantic language representations may be themselves impaired in our 4 WS children. In that case, no normal interactions between STM and long-term language representations would be possible, as the language representations themselves are deficient. This is probably the most parsimonious explanation as it could explain both the impairment in the phonological awareness tasks, and the reduced influence of phonological and lexico-semantic language knowledge on both STM and phonological awareness tasks. Finally, it could be that short-term retention of words and especially non-words in WS is not performed by a phonolog-ically-based STM system, but rather by another more acoustically-based STM system which could be fairly well developed in WS. This suggestion is supported by the fact that performance for LF non-words, in particular, was preserved in our WS children and that differences in performance as a function of linguistic status of the stimuli processed in STM were seriously reduced. Reports describing hyperverbal or 'cocktail party' speech in WS where WS children seem to repeat accurately any verbal information they hear, but without organizing this information in any linguistically meaningful way might support our suggestion (Gosch et al., 1994; Udwin and Yule, 1990). Further studies are needed to explore the importance of acoustic variables on verbal STM processing in WS

\section{Acknowledgements}

Steve Majerus is a Research Fellow at the Fonds National de la Recherche Scientifique (FNRS), Belgium. We are especially grateful to the participants and their families for their time and patience devoted to this study. We thank the two anonymous reviewers for helpful comments on an earlier version of this paper. 
Published in: Neurocase (2003), vol.9, iss.5, pp.390-401

Status: Postprint (Author's version)

\section{References}

Baddeley A. Working memory. Oxford, England, UK: Clarendon Press/Oxford University Press, 1986.

Baddeley A, Gathercole S, Papagno C. The phonological loop as a language learning device. Psychological Review 1998; 105(1): 158-73.

Baddeley AD, Hitch GJ. Working memory. In: Bower GH, editor. The psychology of learning and motivation. San Diego, CA: Academic Press, 1974: 47-90

Barisnikov K, Van der Linden M, Poncelet M. Acquisition of new words and phonological working memory in Williams syndrome: A case study. Neurocase 1996; 2: 395-404.

Bellugi U, Lichtenberger L, Jones W, Lai Z, St George M. The neurocognitive profile of Williams syndrome: A complex pattern of strengths and weakness. Journal of Cognitive Neuroscience 2000; 12(Supplement): 7-29.

Bellugi U, Wang P, Jernigan TL. Williams syndrome: An unusual neuropsychological profile. In: Broman S, Grafman J, editors. Atypical cognitive deficits in developmental disorders: Implications for brain functions. Hillsdale, NJ: Erlbaum, 1994: 23-56.

Bishop DVM. An innate basis for language? Science 1999; 286: 2283-4

Böhning M, Campbell R, Karmiloff-Smith A. Audiovisual speech perception in Williams syndrome. Neuropsychologia 2001; 39: 1-11.

Clahsen H, Almazan M. Syntax and morphology in Williams syndrome. Cognition 1998; 68(3): 167-98.

Content A, Mousty P, Radeau M. BRULEX. Une base de donnees lexicales informatisee pour le francais ecrit et parle./BRULEX: A computerized lexical data base for the French language. Année Psychologique 1990; 90: 551-66.

Crawford JR, Garthwaite PH. Investigation of the single case in neuropsychology: Confidence limits on the abnormality of test scores and test score differences. Neuropsychologia 2002; 40: 1196-208.

Dunn LM, Thériault-Whalen CM, Dunn LM. Echelle de vocabulaire en images Peabody. Adaptation française du Peabody Picture Vocabulary Test. Toronto, Canada: Psycan, 1993.

Ewart AK, Jin W, Atkinson DL, Morris CA, Keating MT Supravulvar aortic stenosis associated with a deletion disrupting the elastin gene Journal of Clinical Investigations 1994; 83: 1071-7.

Frangiskakis JM, Ewart AK, Morris CA, Mervis CB, Bertrand J, Robinson BF, Klein BP, Ensing GJ, Everett LA, Green ED, Pröschel C, Gutowski NJ, Nobie M, Atkinson DL, Odelberg SJ, Keating MT. LIM-Kinase hemi-zygosity implicated in impaired visuospatial cognition. Cell 1996; 86: 59-69.

Gathercole SE, Baddeley AD. Evaluation of the role of phonological STM in the development of vocabulary in children: A longitudinal study. Journal of Memory and Language 1989; 28: 200-13.

Gathercole SE, Baddeley AD. The children's test of non-word repetition. London: The Psychological Corporation 1996.

Gathercole SE, Frankish CR, Pickering SJ, Peaker S. Phonotactic influences on short-term memory. Journal of Experimental Psychology: Human Learning and Memory 1999; 25(1): 84-95.

Gathercole SE, Willis CS, Baddeley AD, Emslie H. The children's test of non-word repetition: A test of phonological working memory. Memory 1994; 2(2): 103-27.

Gosch A, Städing G, Pankau R. Linguistic abilities in children with Williams-Beuren syndrome. American Journal of Medical Genetics 1994; 52: 291-6.

Grant J, Karmiloff-Smith A, Berthoud I, Christophe A. Is the language of people with Williams syndrome mere mimicry? Phonological short-term memory in a foreign language. Cahiers de Psychologie Cognitive/Current Psychology of Cognition 1996; 15(6): 615-28.

Grant J, Karmiloff-Smith A, Gathercole SE, Paterson S, Howlin P, Davies M, Udwin O. Phonological short-term memory and its relationship to language in Williams syndrome. Cognitive Neuropsychiatry 1997; 2(2): 81-99.

Howlin P, Davies M, Udwin O. Syndrome specific characteristics in Williams syndrome: To what extent do early behavioural patterns persist into adult life? Journal of Applied Research in Intellectual Disabilities 1998; 11(3): 207-26.

Hulme C, Maughan S, Brown GD. Memory for familiar and unfamiliar words: Evidence for a long-term memory contribution to short-term memory span. Journal of Memory and Language 1991; 30: 685-701.

Jarrold C, Baddeley AD, Hewes AK. Verbal and nonverbal abilities in the Williams syndrome phenotype: Evidence for diverging developmental trajectories. Journal of Child Psychology and Psychiatry and Allied Disciplines 1998; 39(4): 511-23. 
Published in: Neurocase (2003), vol.9, iss.5, pp.390-401

Status: Postprint (Author's version)

Jarrold C, Baddeley AD, Hewes AK. Genetically dissociated components of working memory: Evidence from Down's and Williams syndrome. Neuropsychologia 1999; 37: 637-51.

Jarrold C, Hartley SJ, Phillips C, Baddeley AD. Word fluency in Williams syndrome: Evidence for unusual semantic organisation? Cognitive Neuropsychiatry 2000; 5(4): 293-319.

Karmiloff-Smith A, Tyler LK, Voice K, Sims K, Udwin O, Howlin P, Davies M. Linguistic dissociations in Williams syndrome: Evaluating receptive syntax in on-line and off-line tasks. Neuropsychologia 1998; 36(4): 343-51.

Laakmann WJ. Cerebellar contributions to cognition: Evidence from Williams syndrome. Dissertation Abstracts International Section B : The Sciences and Engineering 2001; 62(5B): 2490.

Laing E, Hulme C, Grant J, Karmiloff-Smith A. Learning to read in Williams syndrome: Looking beneath the surface of atypical reading development. Journal of Child Psychology and Psychiatry 2001; 42(6): 729-39.

Logie RH, Delia Salla S, Laiacona M, Chalmers P, Wynn V Group aggregates and individual reliability: The case of verbal short-term memory. Memory and Cognition 1996; 24(3): 305-21.

Majerus S, Van der Linden M. LTM effects on verbal STM: A replication study. British Journal of Developmental Psychology 2003; 21: 303-10.

Masquelier MP. Elaboration d'épreuves d'évaluation de la mémoire chez les personnes âgées. Unpublished Mémoire de licence, University of Liège, 1988

Mervis CB, Robinson BF. Expressive vocabulary ability of toddlers with Williams syndrome or Down syndrome: A comparison. Developmental Neuropsychology 2000; 17(1): 111-26.

Morais J, Alegria J, Content A. The relationships between segmental analysis and alphabetical literacy: An interactive view. Cahiers de Psychologie Cognitive/European Bulletin of Cognitive Psychology 1987; 7(5): 415-38.

Nairne JS. A feature model of immediate memory. Memory and Cognition 1990; 18: 251-69.

Paterson SJ, Brown JH, Gsödl MK, Johnson MH, Karmiloff-Smith A. Cognitive modularity and genetic disorders. Science 1999; 286: 2355-

Poirier M, Saint-Aubin J. Immediate serial recall, word frequency, item identity and item position. Canadian Journal of Experimental Psychology 1996; 50: 408-12

Poncelet M, Van der Linden M. Evaluation du stock phonologique de la mémoire de travail: élaboration d'une épreuve de répétition de nonmots pour population francophone. Revue de Neuropsychologie 2003; (in press)

Roodenrys S, Hulme C, Alban J, Ellis AW. Effects of word frequency and age of acquisition on short-term memory span. Memory and Cognition 1994; 22: 695-701.

Service E. The effect of word length on immediate serial recall depends on phonological complexity, not articulatory duration. The Quarterly Journal of Experimental Psychology 1998; 51A(2): 283-304

Sherrets S, Quattrocchi M, Menolascino F Psychological and neuropsychological findings in a child with supravulvar aortic stenosis (Williams syndrome). International Journal of Clinical Neuropsychology 1982; 4(4): 186-90.

Temple CM, Almazan M, Sherwood S. Lexical skills in Williams Syndrome: A cognitive neuropsychological analysis. Journal of Neurolinguistics 2002; 15: 463-95.

Tubach JL, Boë LJ. Un corpus de transcription phonétique. France: Telecom, 1990.

Udwin O, Yule W. Expressive language of children with Williams syndrome. American Journal of Medical Genetics 1990; S6: 108-14.

Vicari S, Brizzolara D, Giovanni AC, Pezzini G. Memory abilities in children with Williams syndrome. Cortex 1996a; 32(3): 503-14.

Vicari S, Carlesimo G, Brizzolara D, Pezzini G. Short-term memory in children with Williams syndrome: A reduced contribution of lexicalsemantic knowledge to word span. Neuropsychologia 1996b; 34(9): 919-25.

Vitevitch MS, Luce PA. When words compete: Levels of processing in perception of spoken words. Psychological Science 1998; 9(4): 3259.

Volterra V, Capirci O, Caselli MC. What atypical populations can reveal about language development: The contrast between deafness and Williams syndrome. Language and Cognitive Processes 2001; 16(2/3): 219-39. 
Published in: Neurocase (2003), vol.9, iss.5, pp.390-401

Status: Postprint (Author's version)

Wang PP, Bellugi U. Evidence from two genetic syndromes for a dissociation between verbal and visual-spatial short-term memory. Journal of Clinical and Experimental Neuropsychology 1994; 16(2): 317-22.

Wechsler D. Echelle d'intelligence pour enfants - révisée. Paris: Centre de Psychologie Appliquée, 1981. 